Pain, Fatigue, and Trauma

\title{
CANCER-RELATED FATIGUE
}

Neurological function figures prominently in essentially all of the 11 symptoms related to cancerrelated fatigue in the proposed diagnostic criteria. Some studies have suggested that quality-of-life measures may be as important as predictors of survival in cancer as is response to chemotherapy.

\section{CHRONIC FATIGUE SYNDROME}

The criteria for diagnosing chronic fatigue syndrome were officially defined by the Centers for Disease Control in 1988 and revised in 2001. The Oxford criteria differ slightly. The British criteria insist on the presence of mental fatigue, although the American criteria include a requirement for several physical symptoms, reflecting the belief that chronic fatigue syndrome has an underlying immune or infectious pathology.

There may be overlaps between individuals satisfying diagnostic criteria for chronic fatigue syndrome and those meeting criteria for possibly related syndromes, such as fibromyalgia.

\section{COMPLEX REGIONAL PAIN SYNDROME}

Complex regional pain syndrome (CRPS) was formerly known as reflex sympathetic dystrophy or causalgia. It is now split into CRPS types I and II. One reason for the change in the nomenclature is that it is not always clear that the pain is mediated by the sympathetic nervous system. However, the new diagnostic criteria for CRPS retain the presence of autonomic changes at some point during the illness evolution, usually early in the course. The major distinguishing feature between the types is whether there is a history of nerve injury. Thus, CRPS type I is synonymous with reflex sympathetic dystrophy (no discrete nerve injury), whereas CRPS type II is synonymous with causalgia (with nerve injury). The injured nerve is generally a large named nerve such as median, sciatic, femoral, and so on. Weakness may be present, in the form of paralysis or dystonia, but is not a diagnostic feature of CRPS.

Modifications and proposed research diagnostic criteria were published in 1999 and are summarized in Tables 7 and 8. The authors found that the above-listed criteria, when used in isolation, lacked specificity and led to overdiagnosis. Using these modifications, diagnostic accuracy rates of about $85 \%$ were achieved for both CRPS and non-CRPS pain syndromes.

\section{FATIGUE IN MULTIPLE SCLEROSIS}

Fatigue in multiple sclerosis has been defined by the 1998 Paralyzed Veterans of America Multiple Sclerosis Council for Clinical Practice Guidelines as "a subjective lack of physical and/or mental energy that is perceived by the individual or caregivers to interfere with usual or desired activities."

Acute fatigue is defined as new or significant increase in fatigue within the last 6 weeks and as a fatigue that limits functional activities or quality of life. 
Table 1

Proposed (1998 Draft) International Statistical Classification of Diseases and Related Health Problems, 10th Edition, Criteria for Cancer-Related Fatigue

Six (or more) of the following symptoms have been present every day or nearly every day during the same 2-week period in the past month, and at least one of the symptoms is (A1) significant fatigue.

A.

1. Significant fatigue, diminished energy, or increased need to rest, disproportionate to any recent change in activity level.

2. Complaints of generalized weakness or limb heaviness.

3. Diminished concentration or attention.

4. Decreased motivation or interest to engage in usual activities.

5. Insomnia or hypersomnia.

6. Experience of sleep as unrefreshing or nonrestorative.

7. Perceived need to struggle to overcome inactivity.

8. Marked emotional reactivity (e.g., sadness, frustration, or irritability) to feeling fatigued.

9. Difficulty completing daily tasks attributed to feeling fatigued.

10. Perceived problems with short-term memory.

11. Postexertional malaise lasting several hours.

B. The symptoms cause clinically significant distress or impairment in social, occupational, or other important areas of functioning.

C. There is evidence from the history, physical examination, or laboratory findings that the symptoms are a consequence of cancer or cancer therapy.

D. The symptoms are not primarily a consequence of comorbid psychiatric disorders, such as major depression, somatization disorder, somatoform disorder, or delirium.

Adapted with permission from Cella D, Peterman A, Passik S, et al. Progress toward guidelines for the management of fatigue. Oncology 1998;12:S369-S377.

Table 2

Centers for Disease Control Criteria for Chronic Fatigue Syndrome

Clinically evaluated, unexplained, persistent, or relapsing fatigue that is:

- Of new or definite onset.

- Not a result of ongoing exertion.

- Not alleviated by rest.

- Results in a substantial reduction in previous levels of occupational, social, or personal activity.

Four or more of the following symptoms that persist or recur during six or more consecutive months of illness and that do not predate the fatigue:

- Self-reported impairment of short-term memory or concentration.

- Sore throat.

- Tender lymph nodes.

- Muscle pain.

- Multijoint pain without swelling or redness.

- Headaches of a new type, pattern, or severity.

- Unrefreshing and/or interrupted sleep.

- Postexertion malaise (a feeling of general discomfort or uneasiness) lasting more than 24 hours.

Exclusion criteria:

1. Any active medical condition that may explain the presence of chronic fatigue, such as untreated hypothyroidism, sleep apnea and narcolepsy, and iatrogenic conditions, such as side effects of medication.

2. Some diagnosable illnesses may relapse or may not have completely resolved during treatment. If the persistence of such a condition could explain the presence of chronic fatigue, and if it cannot be clearly established that the original condition has completely resolved with treatment, then such patients should not be classified as having chronic fatigue syndrome. Examples of illnesses that can present such a picture include some types of malignancies and chronic cases of hepatitis B or C virus infection.

3. Any past or current diagnosis of a major depressive disorder with psychotic or melancholic features such as: a. Bipolar affective disorders.

b. Schizophrenia of any subtype. 
Table 2 (Continued)

c. Delusional disorders of any subtype.

d. Dementias of any subtype.

e. Anorexia nervosa.

f. Bulimia nervosa.

4. Alcohol or other substance abuse, occurring within 2 years of the onset of chronic fatigue and any time afterwards.

5. Severe obesity as defined by a body mass index (body mass index = weight in kilograms/[height in meters] $\times 2$ ) equal to or greater than 45 . (Note: Body mass index values vary considerably among different age groups and populations. No "normal" or "average" range of values can be suggested in a meaningful fashion. The range of 45 or greater was selected because it falls clearly within the range of severe obesity.)

Adapted from the website of the Centers for Disease Control and Prevention. Available at http://www.cdc.gov/ncidod/ diseases/cfs/index.htm.

Table 3

Oxford (British) Criteria for Chronic Fatigue Syndrome

Severe disabling fatigue of at least a 6-month duration that:

- Affects both physical and mental functioning.

- Is present for more than $50 \%$ of the time.

Other symptoms, particularly myalgia and sleep and mood disturbances, may be present.

Exclusion criteria:

- Active, unresolved, or suspected disease that is likely to cause fatigue.

- Psychotic, melancholic, or bipolar depression (but not uncomplicated major depression).

- Psychotic disorders.

- Dementia.

- Anorexia or bulimia nervosa.

Adapted from Archard L, Banatvala JE, et al. A report-chronic fatigue syndrome: guidelines for research. J R Soc Med 1991;84:118-121.

Table 4

Additional Symptoms Frequent Among Patients With Chronic Fatigue Syndrome

Although the symptoms heretofore listed are the official diagnostic criteria, many patients with chronic fatigue syndrome present a variety of other symptoms, including:

- Pain (almost universal in chronic fatigue).

- Allergies.

- Chemical sensitivities.

- Secondary infections, including Candida and viral infections.

- Cognitive impairment, including short-term memory loss, difficulty concentrating and doing word searches and math problems.

- Digestive disturbances, such as chronic constipation or diarrhea.

- Night sweats or spontaneous daytime sweats, unaccompanied by fever.

- Headaches, migraines.

- Weakness (paresis), muscle fatigue, and pain (fibromyalgia).

- Premenstrual syndrome.

- Sleep disorders, including excessive sleep (hypersomnia), light sleep, or an inability to sleep for more than an hour (hyposomnia), disturbing nightmares.

- A period of 1-3 hours after awakening, during which patients are too exhausted to get out of bed.

- Cystitis (inflammation of the urinary bladder), particularly interstitial cystitis in which urine cultures are negative.

- Vision and eye problems, including sensitivity to light (photophobia), dry eyes, tunnel vision, night blindness, and difficulty focusing.

An initial office examination may also find the following signs:

- Low blood pressure, particularly on standing (orthostatic hypotension). 
Table 4 (Continued)

- Low oral temperatures (less than $97^{\circ} \mathrm{F}$ ).

- Slightly elevated oral temperatures (but less than $100^{\circ} \mathrm{F}$ ), which are part of persistent flu-like symptoms.

- Increased heart rate (tachycardia).

- A positive Romberg test (unsteadiness when standing with eyes closed).

Adapted from the website of the Centers for Disease Control and Prevention. Available at http://www.cdc.gov/ncidod/ diseases/cfs/index.htm.

Table 5

Tests for Screening of Chronic Fatigue Syndrome Laboratory Tests for Evaluation of Possible Chronic Fatigue Syndrome

- Complete blood count with differential.

- Complete metabolic panel.

- Erythrocyte sedimentation rate.

- Urinalysis.

Optional tests include:

- Antinuclear antibodies and rheumatoid factor.

- Thyroid tests (T3, T4, thyroid-stimulating hormone).

- Adrenal tests (AM and PM cortisol levels).

- Lyme titers and HIV serology.

Specific tests that support (but do not necessarily confirm) a diagnosis of chronic fatigue include:

- Tests for viral infections, such as cytomegalovirus, Epstein-Barr virus, human herpesvirus 6, and Coxsackie virus.

- Immune system tests, including low natural-killer cell counts, elevated interferon- $\alpha$, tumor necrosis- $\alpha$, interleukins 1 and 2, T-cell activation, altered T4/T8 cell ratios, low T-cell suppressor cell count, fluctuating B- and T-cell counts, antinuclear antibodies, immunoglobulin deficiency, and antithyroid antibodies.

- Exercise testing may show decreased cortisol levels after exercise, decreased cerebral blood flow after exercise, inefficient glucose utilization, and erratic breathing patterns.

Adapted from the website of the Centers for Disease Control and Prevention. Available at http://www.cdc.gov/ncidod/ diseases/cfs/index.htm.

Table 6

Diagnostic Criteria for Complex Regional Pain Syndrome

I. Type I (reflex sympathetic dystrophy)

A. The presence of an initiating noxious event, or a cause of immobilization.

B. Ongoing spontaneous pain, allodynia, or hyperalgesia is disproportionate to any inciting event and is not limited to the distribution of a single peripheral nerve.

C. Evidence at some time of autonomic dysfunction, such as edema, changes in skin blood flow, hyperhidrosis, or abnormal sudomotor activity in the region of the pain.

D. The diagnosis is excluded by the existence of conditions that would otherwise account for the degree of pain and dysfunction.

II. Complex regional pain syndrome type II (causalgia)

A. Type II is a syndrome that develops after a nerve injury. Spontaneous pain or allodynia/hyperalgesia occurs and is not necessarily limited to the territory of the injured nerve.

B. There is or has been evidence of edema, skin blood-flow abnormality, or abnormal sudomotor activity in the region of the pain since the inciting event.

C. This diagnosis is excluded by the existence of conditions that would otherwise account for the degree of pain and dysfunction.

Adapted from Stanton-Hicks M, Janig W, Hassenbusch S, Haddox JD, Boas R, Wilson P. Reflex sympathetic dystrophy: changing concepts and taxonomy. Pain 1995;63:127-133, with permission from Elsevier. 
Table 7

Signs and/or Symptoms on Complex Regional Pain Syndrome Checklist

1. "Burning" pain.

2. Hyperesthesia.

3. Temperature asymmetry.

4. Color changes.

5. Sweating changes.

6. Edema.

7. Nail changes.

8. Hair changes.

9. Skin changes.

10. Weakness.

11. Tremor.

12. Dystonia.

13. Decreased range of motion.

14. Hyperalgesia.

15. Allodynia.

Adapted from Bruehl S, Harden RN, Galer BS, et al. External validation of IASP diagnostic criteria for complex regional pain syndrome and proposed research diagnostic criteria. Pain 1999;81:147-154.

Table 8

Proposed Modified Research Diagnostic Criteria for Complex Regional Pain Syndrome

1. Continuing pain that is disproportionate to any inciting event.

2. Must report at least one symptom in each of four following categories:

a. Sensory

i. Reported hyperesthesia.

b. Vasomotor

i. Temperature asymmetry.

ii. Skin color changes.

iii. Skin color asymmetry.

c. Sudomotor/edema

i. Edema.

ii. Sweating changes.

iii. Sweating asymmetry.

d. Motor/trophic

i. Decreased range of motion.

ii. Motor dysfunction

(1) Weakness.

(2) Tremor.

(3) Dystonia.

i. Trophic changes

(1) Skin.

(2) Nails.

(3) Hair.

3. Must display at least one sign in two or more of the following categories:

a. Sensory

i. Hyperalgesia to pinprick.

ii. Allodynia to light touch.

iii. Vasomotor

(1) Evidence of temperature asymmetry.

(2) Evidence of skin color changes or asymmetry.

b. Sudomotor/edema

i. Evidence of edema.

ii. Evidence of sweating changes.

iii. Evidence of sweating asymmetry. 
Table 8 (Continued)

c. Motor/trophic

i. Evidence of decreased range of motion.

ii. Evidence of motor dysfunction

(1) Tremor.

(2) Weakness.

(3) Dystonia.

d. Evidence of trophic changes

i. Skin.

ii. Nails.

iii. Hair.

Adapted from Bruehl S, Harden RN, Galer BS, et al. External validation of IASP diagnostic criteria for complex regional pain syndrome and proposed research diagnostic criteria. Pain 1999;81:147-154, with permission from Elsevier.

Chronic persistent fatigue is defined as fatigue that is present for any amount of time on $50 \%$ of the days for 6 weeks and fatigue that limits functional activities or quality of life.

\section{FIBROMYALGIA}

\section{Table 9}

\section{Criteria for the Classification of Fibromyalgia}

1. History of widespread pain

Definition: Pain is considered widespread when all of the following are present: pain in the left side of the body, pain in the right side of the body, pain above the waist, and pain below the waist. In addition, axial skeletal pain (cervical spine or anterior chest or thoracic spine or low back) must be present. In this definition, shoulder and buttock pain is considered as pain for each involved side. "Low back pain" is considered lower-segment pain.

2. Pain in 11 of 18 tender point sites on digital palpation

Definition: Pain, on digital palpation, must be present in at least 11 of the following 18 sites:

Occiput: bilateral, at the suboccipital muscle insertions.

Low cervical: bilateral, at the anterior aspects of the intertransverse spaces at C5-C7.

Trapezius: bilateral, at the midpoint of the upper border.

Supraspinatus: bilateral, at origins above the scapula spine near the medial border.

Second rib: bilateral, at the second costochondral junctions, just lateral to the junctions on upper surfaces.

Lateral epicondyle: bilateral, $2 \mathrm{~cm}$ distal to the epicondyles.

Gluteal: bilateral, in upper outer quadrants of buttocks in anterior fold of muscle.

Greater trochanter: bilateral, posterior to the trochanteric prominence.

Knee: bilateral, at the medial fat pad proximal to the joint line.

Digital palpation should be performed with an approximate force of $4 \mathrm{~kg}$.

For a tender point to be considered "positive," the subject must state that the palpation was painful. "Tender" is not to be considered "painful."

For classification purposes, patients will be said to have fibromyalgia if both criteria are satisfied. Widespread pain must have been present for at least 3 months. The presence of a second clinical disorder does not exclude the diagnosis of fibromyalgia.

(Adapted from Wolfe F, Smythe HA, Yunus MB, et al. The American College of Rheumatology 1990 criteria for the classification of fibromyalgia: report of the multicenter criteria committee. Arthritis Rheum 1999;33:160-172, with permission from John Wiley and Sons, Inc.)

\section{MINOR HEAD INJURY}

Despite being one of the most common conditions involving trauma and the nervous system, there remains wide variability in diagnostic criteria for minor head trauma. One method of categorizing head trauma is by using the initial Glasgow Coma Scale. Minimal head injury corresponds to a score of 15, without loss of consciousness. A score of 14 to 15, with loss of consciousness lasting less than 5 minutes, constitutes mild head injury and no neurological deficit. Those with scores of 13 would be considered 
Table 10

Diagnostic Criteria for Minor Head Injury

\begin{tabular}{lrc}
\hline Criterion & $n$ & Percentage \\
\hline Loss of consciousness & 58 & 82 \\
Posttraumatic amnesia & 30 & 42 \\
Impaired level of consciousness & 5 & 7 \\
Absence of focal neurological deficit $^{\text {Other signs and symptoms }}{ }^{a}$ & 6 & 8 \\
No definition & 29 & 41 \\
\hline
\end{tabular}

In all, 64 hospitals listed which diagnostic criteria they used to define minor head injury.

${ }^{a}$ Vertigo, nausea, vomiting, headache, confusion.

(Adapted with permission from Bellner J, Jensen S-M, Romner B. Diagnostic criteria and use of ICD-10 codes to define and classify minor head injury. J Neurol Neurosurg Psychiatry 2003;74:351-352.)

\section{Table 11}

Recommended Definitions for Mild Traumatic Brain Injury

\section{Incident cases of mild traumatic brain injury (MTBI)}

The conceptual definition of MTBI is an injury to the head as a result of blunt trauma or acceleration or deceleration forces that result in one or more of the following conditions:

- Any period of observed or self-reported:

o Transient confusion, disorientation, or impaired consciousness.

o Dysfunction of memory around the time of injury.

o Loss of consciousness lasting less than 30 minutes.

- Observed signs of neurological or neuropsychological dysfunction, such as:

o Seizures acutely following injury to the head.

o Among infants and very young children: irritability, lethargy, or vomiting following head injury.

o Symptoms among older children and adults such as headache, dizziness, irritability, fatigue, or poor concentration, when identified soon after injury, can be used to support the diagnosis of MTBI, but cannot be used to make the diagnosis in the absence of loss of consciousness or altered consciousness. Research may provide additional guidance in this area.

Based on this conceptual definition, separate operational definitions of MTBI are recommended for cases identified from interviews and surveys, administrative health care datasets, and patient medical records.

Adapted from Center for Disease Control and Prevention, National Center for Injury Prevention and Control. Report to Congress on mild traumatic brain injury in the United States: steps to prevent a serious public health problem. September 2003. Available at http://www.cdc.gov/Migrated_Content/Report/TBI_Report_to_Congress_on_MTBI_ Sept_2003.pdf.

to have moderate head injury. However, the experience in the field of athletic injuries, especially as related to "concussion," indicates that loss of consciousness may be hard to discern and of variable import in injury severity.

In a survey of Swedish hospitals, Bellner et al. found wide variability in application of head injury diagnostic criteria, as indicated by International Statistical Classification of Diseases and Related Health Problems, 10th Edition, codes (Table 10).

In a report to the United States Congress, a committee reported on the epidemiology and prevention of mild head trauma. Their operational definition is presented in Table 11.

\section{PAIN DISORDERS}

The International Association for the Study of Pain defines pain as "an unpleasant sensory and emotional experience associated with actual or potential tissue damage, or described in terms of such damage."

Several aspects of this definition are worth noting. Pain is linked to the concept of consciousness, in terms of the words "unpleasant experience." This definition also puts the concept of pain squarely in terms 
Table 12

Diagnostic Criteria for Pain Disorder

- Pain in one or more anatomic sites is the predominant focus of the clinical presentation and is of sufficient severity to warrant clinical attention.

- The pain causes clinically significant distress or impairment in social, occupational, or other important areas of functioning.

- Psychological factors are judged to have an important role in the onset, severity, exacerbation, or maintenance of the pain.

- The symptom or deficit is not intentionally produced or feigned (as in factitious disorder or malingering).

- The pain is not better accounted for by a mood, anxiety, or psychotic disorder and does not meet criteria for dyspareunia.

\section{Code as follows:}

Pain disorder associated with psychological factors: Psychological factors are judged to have the major role in the onset, severity, exacerbation, or maintenance of the pain. (If a general medical condition is present, it does not have a major role in the onset, severity, exacerbation, or maintenance of the pain.) This type of pain disorder is not diagnosed if criteria are also met for somatization disorder.

\section{Specify if:}

Acute: duration of less than 6 months.

Chronic: duration of 6 months or longer.

Pain disorder associated with both psychological factors and a general medical condition: Both psychological factors and a general medical condition are judged to have important roles in the onset, severity, exacerbation, or maintenance of the pain. The associated general medical condition or anatomic site of pain is coded on Axis III.

\section{Specify if:}

Acute: duration of less than 6 months.

Chronic: duration of 6 months or longer.

Adapted from American Psychiatric Association. Diagnostic and Statistical Manual of Mental Disorders, 4th rev. ed. Washington, DC: American Psychiatric Association, 1994.

\section{Table 13}

\section{Diagnostic Criteria for Second-Impact Syndrome}

\begin{tabular}{ll}
\hline Criteria & Definition \\
\hline (a) & Medical review after a witnessed first impact \\
(b) & Documentation of ongoing symptoms following the first impact \\
up to the time of the second impact \\
(c) \\
Witnessed second head impact with a subsequent rapid \\
cerebral deterioration \\
Neuropathological or neuroimaging evidence of cerebral swelling without \\
significant intracranial hematoma or other cause for edema
\end{tabular}

Definite second-impact syndrome (SIS): (a), (b), (c), and (d).

Probable SIS: (c) and (d) and either (a) or (b).

Possible SIS: (c) and (d) only.

Not SIS: (c) or (d) absent.

Adapted from McCrory PR, Berkovic SF. Second-impact syndrome. Neurology 1998;50:677-683, with permission from Lippincott, Williams, and Wilkins.

of the patient's perception. It does not specify any objective correlate in terms of physiological functioning or evidence of tissue damage. Understanding the concept may help some trainees and practitioners in evaluation of individuals presenting with pain. Implicit in the diagnosis is a central component of pain, whose biological basis involves multiple levels of the neuraxis and inputs from multiple neurotransmitter systems. However, the definition does not specify how this may change as related to the chronicity of the pain.

Several discrete pain syndromes have been described and are listed in Table 12. 


\section{SECOND-IMPACT SYNDROME}

A syndrome of diffuse cerebral swelling with catastrophic results after a second head injury. It is seen most commonly in children and adolescents, particularly in the setting of sports injuries with a brief concussion, when a second impact occurs before the symptoms of the first injury have cleared, resulting in more severe cerebral injury.

\section{SOURCES}

\section{Cancer-Related Fatigue}

Cella D, Peterman A, Passik S, et al. Progress toward guidelines for the management of fatigue. Oncology 1998;12:S369-S377.

Cella D, Davis K, Breitbart W, et al. Cancer-related fatigue: prevalence of proposed diagnostic criteria in a United States sample of cancer survivors. J Clin Oncol 2001;19:3385-3391.

\section{Chronic Fatigue Syndrome}

Fukuda K, Straus SE, Hickey I, et al. The chronic fatigue syndrome: a comprehensive approach to its definition and study. Ann Intern Med 1994;121:953-959.

Holmes G, Kaplan J, Gantz N, et al. Chronic fatigue syndrome: a working case definition. Ann Intern Med 1988;108:387-389.

Reeves WC, Lloyd A, Vernon SD, et al. Identification of ambiguities in the 1994 chronic fatigue syndrome research case definition and recommendations for resolution. BMC Health Serv Res 2003;3:25.

Sharpe MC, Archard L, Banatvala JE, et al. A report-chronic fatigue syndrome: guidelines for research. J R Soc Med 1991;84:118-121.

\section{Complex Regional Pain Syndrome}

Bruehl S, Harden RN, Galer BS, et al. External validation of IASP diagnostic criteria for complex regional pain syndrome and proposed research diagnostic criteria. Pain 1999;81:147-154.

Hassantash SA, Afrakhteh M, Maier RV. Causalgia: a meta-analysis of the literature. Arch Surg 2003;138:1226-1231.

Stanton-Hicks M, Janig W, Hassenbusch S, Haddox JD, Boas R, Wilson P. Reflex sympathetic dystrophy: changing concepts and taxonomy. Pain 1995;63:127-133.

\section{Fatigue in Multiple Sclerosis}

MS Council for Clinical Practice Guidelines. Fatigue in Multiple Sclerosis. Washington, DC: Paralyzed Veterans Association, 1998.

\section{Fibromyalgia}

Wolfe F, Smythe HA, Yunus MB, et al. The American College of Rheumatology 1990 criteria for the classification of fibromyalgia: report of the multicenter criteria committee. Arthritis Rheum 1990;33:160-172.

\section{Minor Head Injury}

Bellner J, Jensen S-M, Romner B. Diagnostic criteria and use of ICD-10 codes to define and classify minor head injury. J Neurol Neurosurg Psychiatr 2003;74:351- 352.

Centers for Disease Control and Prevention, National Center for Injury Prevention and Control. Report to Congress on mild traumatic brain injury in the United States: steps to prevent a serious public health problem. September 2003. Available via http://www.cdc.gov/Migrated_Content/Report/TBI_Report_to_Congress_on_MTBI_Sept_2003.pdf).

\section{Pain Disorders}

American Psychiatric Association. Diagnostic and Statistical Manual of Mental Disorders, 4th rev. ed. Washington, DC: American Psychiatric Association, 1994.

Fishbain DA, Goldberg M, Meagher BR, Steele R, Rosomoff H. Male and female chronic pain patients categorized by DSM-III psychiatric diagnostic criteria. Pain 1986;26:181-197.

\section{Second-Impact Syndrome}

McCrory PR, Berkovic SF. Second impact syndrome. Neurology 1998;50:677-683. 